\title{
Incentive Model and Strategy Research of New Service Development Network Organization Based on Synergetic Perspective
}

\author{
Bu Xinyi ${ }^{1, a}$, Dong Ying ${ }^{2, b}$ \\ ${ }^{1}$ Hangzhou Dianzi University, Hangzhou 310018, China \\ ${ }^{2}$ Hangzhou Dianzi University, Hangzhou 310018, China \\ abxy002@126.com, ${ }^{b}$ bluerose0617@126.com
}

\begin{abstract}
With the increasing of external dependency, successful new service development (NSD) becomes more difficult. This study combines new service development theory and graph theory to analyze agents of new service development network organization. Sequentially, quantitative incentive model is established. Conclusively, numerical simulation analysis contributes to get constructive incentive strategies. Research results suggest that according to the dynamic change of unstable factors in external environment, network organization itself and external agents, adjusting incentive coefficient reasonably will be conducive to promote the performance.
\end{abstract}

Keywords: New Service Development Network Organization; Incentive Model; Strategy Research; Synergetic Perspective

\section{Introduction}

Economic globalization and informatization has intensified uncertainty of service corporations' external environment, which makes market environment and customer demand become more complicated and inconstant [1]. Thus there are a series of difficulties, when service corporations try to meet the rapidly changing market demand only depending on the internal limited resources [2-4]. On the one hand, rapidly changing and high uncertainty in external environment have high requirement on the flexibility and adaptability of service corporations. On the other hand, as service itself has intangibility, heterogeneity, inseparability and fugitiveness [5], service products present various defects, such as short life cycle, etc. Obviously, these difficulties make the process of service innovation fill with serious challenges [6]. As an important research domain of service innovation, new service development (NSD) open a new way for service corporations to innovate.

With the dynamic change of customers' needs, constantly improving the innovation ability and success rate of service corporations is very necessary [7]. Aiming to deal with dynamic change of market demand in NSD process, this research consider the networked interactive synergy between NSD corporations 
and external agents as network organization relationship to study synergetic operation problems. And the agents will be able to accommodate to complex and uncertain environment. In order to assure good function of network organization, governance mechanism is the core issue of governance research [8]. As a consequence, to keep the network organization stable and synergistic operation has important theoretical significance and practical significance.

\section{Definition of NSD Network Organization Structure}

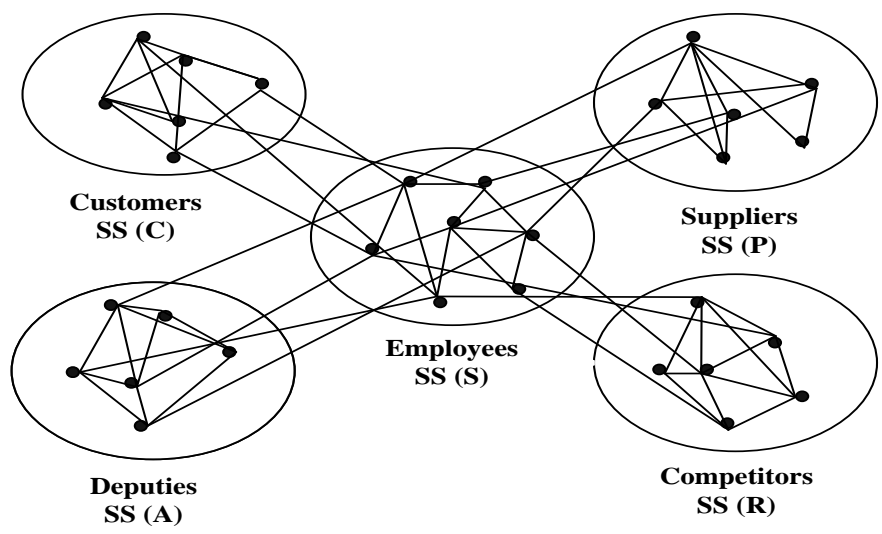

Fig.1. Structure diagram of NSD network organization. ${ }^{\text {a }}$

a synergistic subgroup (SS)

NSD corporations are the core agent (leader) [9], and network organization has the ability to produce synergies under its coordination and guidance. The employees of NSD corporations as nodes keep various contacts with external agents and share information. In addition, the external agents of NSD corporations including customers, suppliers, deputies and competitors. Employees establish connections (edge) with external agents through the exchange of information resources, etc, constituting a network organization that has a complex network structure among five synergistic subgroups (node). The descriptions of these relationships are shown in Fig.1.

\section{Modeling and Simulation Analysis.}

Based on above NSD network organization structure, this study build an incentive model about the degree of information sharing, and design a remuneration allocation mechanism to stimulate the enthusiasm of agents to share information. The purpose is to achieve win-win and produce synergistic effect. From the perspective of information sharing, we outline assumptions as follows.

Assumption 1. Due to the asymmetry of information, NSD corporations 
cannot directly measure the synergistic effort of external agents. The degree of this synergistic effort is denoted by $a$, and let $N=\left(a_{1}, a_{2}, \ldots, a_{n}\right)$ denote $\mathrm{N}$ dimensional vector of synergistic effort based on information sharing.

Assumption 2. The synergistic effort cost of agents is denoted as $C$. We assume that the synergistic effort cost is $C\left(a_{i}\right)=\frac{1}{2} b_{i} a_{i}^{2}(i=1,2, \ldots n)$, and let $b_{i}(i=1,2, \ldots n)$ denote synergistic effort cost coefficient. In addition, the higher the value of $b_{i}$, the higher the cost of per effort.

Assumption 3. We assume that synergistic total benefit of NSD network organization is $S=\lambda \alpha \sum_{i=1}^{n} f\left(a_{i}, e_{i}\right)+\theta$ and the size of $\lambda(0<\lambda<1)$ is negatively related to the negative effects; the cooperation effect coefficient of network organization is denoted as $\alpha$, and it is positively related to $S$; we refer to $f\left(a_{i}, e_{i}\right)=e_{i} a_{i}+\theta_{i}$ as the revenue function, and let $e_{i}$ denote the separate yield rate, in addition, $\theta_{i}$ denotes the external uncertainties of $S$, then we assume that $\theta_{i}(i=1,2, \ldots, n)$ are mutually independent and they obey normal distribution that is $\theta_{i} \sim\left(0, \sigma^{2}\right)$.

Assumption 4. NSD corporations set synergy incentive strategies for the external agents based on the synergistic total benefit. We assume that the synergistic reward of external agent " $i$ " is $P_{i}(S)=\beta_{i} S$, and $\beta_{i}$ in this formula is the corresponding external incentive coefficient. And NSD corporations are risk neutral and the agents are risk avoidant. Moreover, $d_{i}$ is the degree of risk aversion, and its risk cost is $C_{i}=\frac{1}{2} d_{i} \operatorname{Var}\left(P_{i}\right)=\frac{1}{2} d_{i} \operatorname{Var}\left(\beta_{i} S\right)=\frac{1}{2} d_{i} \beta_{i}^{2} \sigma^{2}$.

Alternatively, we refer to $R$ as the total benefit of network organization and $F$ as the total fixed remuneration of external agents from NSD corporations. so the expected profit is:

$E(U)=E\left(R-F+S-\sum_{i=1}^{n} \beta_{i} S\right)=R-F+\lambda \alpha \sum_{i=1}^{n} e_{i} a_{i}-\sum_{i=1}^{n} \beta_{i}\left(\lambda \alpha \sum_{i=1}^{n} e_{i} a_{i}\right)$

In addition, let $F_{i}$ denote as fixed net pay, then, the expected revenue of external agents " $i$ " is

$E\left(V_{i}\right)=E\left(F_{i}+\beta_{i} S-C\left(a_{i}\right)-C_{1}\right)=F_{i}+\beta_{i} \lambda \alpha \sum_{i=1}^{n} e_{i} a_{i}-\frac{1}{2} b_{i} a_{i}^{2}-\frac{1}{2} d_{i} \beta_{i}^{2} \sigma^{2}$. 
Next, we refer to as the reservation utility of external agent “ $i$ ”, and NSD corporations utilize the following incentive model to motivate the network organization.

$\operatorname{Max} E(U)_{\beta_{i}}=R-F+\lambda \alpha \sum_{i=1}^{n} e_{i} a_{i}-\sum_{i=1}^{n} \beta_{i}\left(\lambda \alpha \sum_{i=1}^{n} e_{i} a_{i}\right)$.

S.t. $\operatorname{MaxE}\left(V_{i}\right)=F_{i}+\beta_{i} \lambda \alpha \sum_{i=1}^{n} e_{i} a_{i}-\frac{1}{2} b_{i} a_{i}^{2}-\frac{1}{2} d_{i} \beta_{i}^{2} \sigma^{2} \quad(I C)$.

$E\left(V_{i}\right)=F_{i}+\beta_{i} \lambda \alpha \sum_{i=1}^{n} e_{i} a_{i}-\frac{1}{2} b_{i} a_{i}^{2}-\frac{1}{2} d_{i} \beta_{i}^{2} \sigma^{2} \geq K_{i} \quad(I R)$

Finally, we can obtain the first derivative of $\beta_{i}$ :

$\beta_{i}=\frac{1}{1+b_{i} d_{i} \sigma^{2} / \lambda^{2} \alpha^{2} e_{i}^{2}}$.

In NSD network organization incentive model, there are three kinds of parameters that affect incentive coefficient $\beta_{i}$, that is, the instability factors of external environment ( $d_{i}$ and $\sigma^{2}$ ), the factors of network organization itself ( $\lambda$ and $\alpha$ ), and the factors of external agents $\left(b_{i}\right.$ and $e_{i}$ ). Furthermore, this paper discusses the influence of above variables on incentive coefficient.
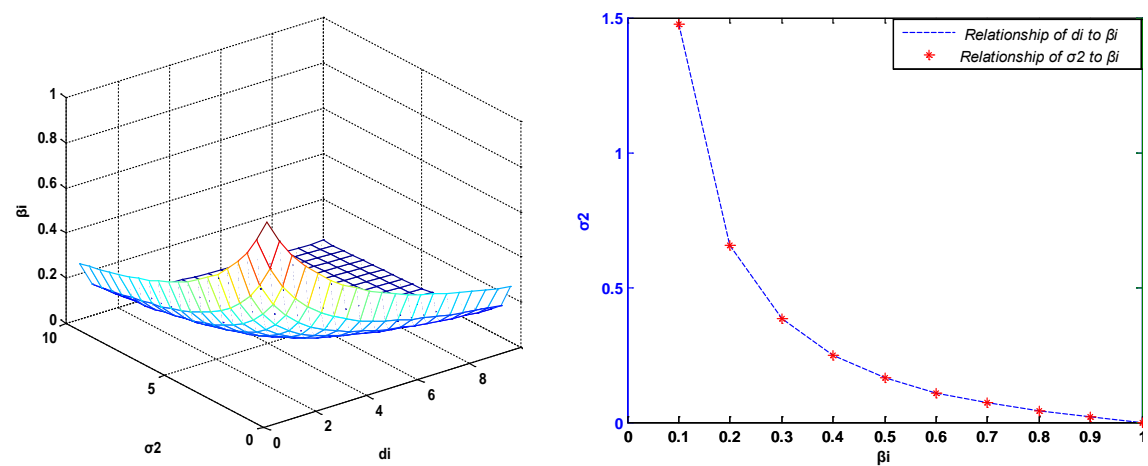

Fig. 2. Relationship among $\beta_{i}, d_{i}$ and $\sigma^{2} \quad$ Fig. 3. Relationship of $d_{i}$ to $\beta_{i}$ and $\sigma^{2}$ to $\beta_{i}$ 

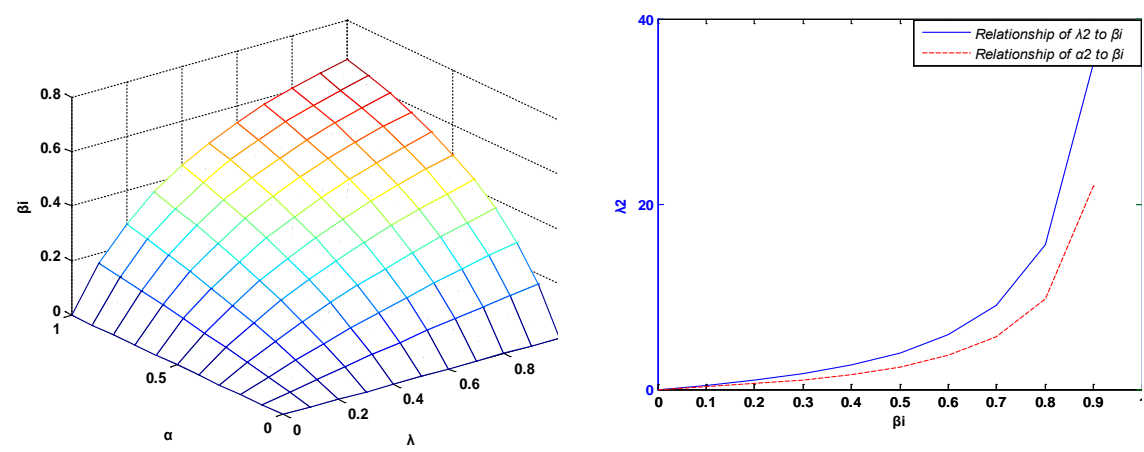

Fig. 4. Relationship among $\beta_{i}, \lambda$ and $\alpha \quad$ Fig. 5. Relationship of $\lambda^{2}$ to $\beta_{i}$ and $\alpha^{2}$ to $\beta_{i}$

(I) If $d_{i}$ and $\sigma^{2}$ are unknown, we set $\lambda=0.8, \alpha=2, b_{i}=1$, $e_{i}=0.8$.

From Fig. 2, the optimal $\beta_{i}$ is significantly negative related to $d_{i}$ and $\sigma^{2}$, furthermore, the less the $d_{i}$ and $\sigma^{2}$, the greater the $\beta_{i}$. When the value of $d_{i}$ and $\sigma^{2}$ is relatively small, NSD corporations can effectively motivate the external agents and improve their efforts.

From Fig. 3. the unilateral influence degrees of $d_{i}$ and $\sigma^{2}$ on $\beta_{i}$ are basically identical. With the increase of $\beta_{i}$, the sensitivities of $\beta_{i}$ to $d_{i}$ and $\sigma^{2}$ present a trend: first increases then decreases. Consequently, in the environment with high-risk and high uncertainty, external agents correspondingly decrease their efforts to get slightly lower but steady benefit.

(II) If $\lambda$ and $\alpha$ are unknown, we set $d_{i}=1, \sigma^{2}=10, b_{i}=1$, $e_{i}=0.8$.

From Fig. 4 , the optimal $\beta_{i}$ is positive related to $\lambda$ and $\alpha$, but the correlation is not big. When $\lambda=\alpha=1$, the value of $\beta_{i}$ is about 0.6 , and with the increase of $\lambda$ and $\alpha$, the correlation of the parameters above is decreasing. It reflects that the network organization is difficult to produce good synergistic effects and NSD network organization needs good governance of NSD corporations.

From Fig. 5, the influence of $\lambda^{2}$ to $\beta_{i}$ is greater than $\alpha^{2}$, and this further reflect the difficulty to achieve synergies. When synergistic effect is relatively high, the $\beta_{i}$ is extremely high. It is obvious that synergistic effect has important significance on the entire network organization. 
(III) If $b_{i}$ and $e_{i}$ are unknown, set $d_{i}=1, \sigma^{2}=10, \lambda=0.8, \alpha=2$.

From Fig. 6 , the optimal $\beta_{i}$ is dynamically affected by $b_{i}$ and $e_{i}$. When $e_{i}$ is relatively big, $b_{i}$ to $\beta_{i}$ is prominent; when $b_{i}$ is relatively small, the influence of $e_{i}$ to $\beta_{i}$ is relatively prominent. Consequently, it is essential to separately investigate the yield rates of external agents.

In Fig. 7, when $e_{i}^{2}$ is greater than the corresponding $e_{i}^{2}$ of intersection, the influence of $e_{i}^{2}$ to $\beta_{i}$ is relatively prominent; when $b_{i}$ is smaller than the corresponding $b_{i}$ of intersection, the influence of $b_{i}$ to $\beta_{i}$ is relatively prominent. Therefore, NSD corporations should separately calculate the profitability of external agents and prioritize them according to the profitability.

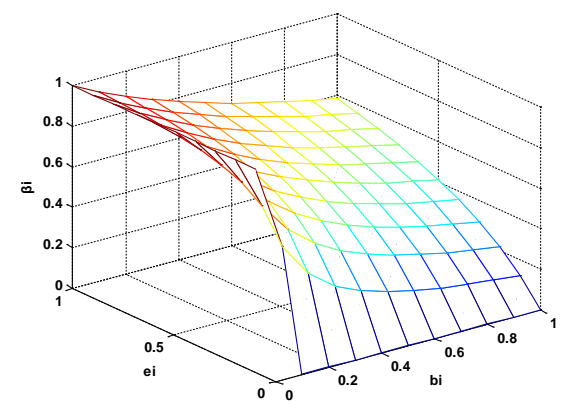

Fig. 6. Relationship among $\beta_{i}, b_{i}$ and $e_{i}$ $\beta_{i}$ and $e_{i}^{2}$ to $\beta_{i}$

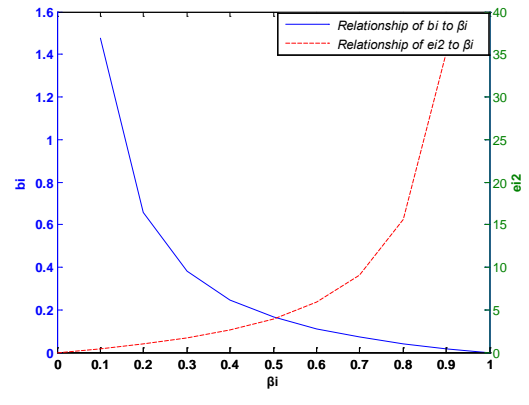

Fig. 7. Relationship of $b_{i}$ to

\section{The Suggestion}

Based on above analysis, we can get the following reasonable NSD network organization incentive strategies.

(I) When risk degree and benefit uncertainty from external environment is relatively high, NSD corporations should reduce incentive coefficient. Simultaneously, NSD corporations ought to create a good external environment.

(II) NSD corporations need to adjust incentive coefficient appropriately. Given the simulation results, it reflects not only the complexity of network organization and the multi-faceted of factors, but also the difficulties to achieve synergies. Then, the high sensitivity of incentive coefficient to cooperative effect indicates that each agent should cooperate, and then achieve win-win-win goals.

(III) NSD corporations ought to give higher incentive to the external agents 
with high $e_{i} / b_{i}$. The function of yield rate is greater than cost coefficient's, and it is necessary to establish a selection and evaluation system of network agents. NSD corporations should prioritize values by evaluation system, and give relatively high benefits to the agents with high-grade and improve the operation efficiency. Apparently, the agents with high quality are the necessary conditions for the good synergistic effect.

(IV) NSD corporations should motivate external agents from several aspects. If they put information sharing into incentive strategies, they will get better results. But in actual economic activity, information sharing mechanism is not as simple as the model, and need to take several auxiliary measures. They need to establish a reliable information sharing system, assess each member prior to accession and consider their reputation. Moreover, fixed benefit distribution should consider effort level and risk degree. After comprehensively measuring, NSD corporations need to review them according to the information sharing mechanism model. If either of the two parameters above does not meet the contract in advance and then network agents have opportunistic behavior, the fixed interest distribution will also get some punishment.

\section{Conclusions}

The research on NSD has broken through the traditional research on NSD organization, concluding the conclusions of two aspects: On the one hand, we regard the external network relationship of NSD as a network organization and define the concept of NSD network organization and its external agents. On the other hand, we introduce both the negative effect coefficient and the cooperation effect coefficient into incentive model to establish an incentive model which is suited to the characteristics of NSD; moreover, we put forward four incentive strategies to promote the synergistic operation of NSD network organization. What's more, we also utilize information bias function and deposit system to obtain information sharing and benefit distribution strategies.

\section{Acknowledgements}

This work was financially supported by the Zhejiang province philosophy social sciences planning project (13NDJC038YB).

\section{References}

[1] M. Luis and M. Camarinha, Collaborative networked organizations: Status and trends in manufacturing, Annual Reviews in Control, vol. 33(2), 2009, p. 199-208.

[2] M. Smith, M. Fischbacher and F. A. Wilson, New service development: from panoramas to precision, European Management Journal, vol. 25(5), 2007, p. 370-383.

[3] Z. Shu-kuan, H. Tao-qun and L. Jin-jin, Enterprise ecological system 
evolution analysis based on logistic model, Journal of Industrial Technological Economics, vol. 27(10), 2008, p. 70-72.

[4] D. Zhenguo, T. Wei and W. Chen, Incentive Model for Resources Sharing, 2011 International Conference on Computer Science and Service System (CSSS), 2011,p.1330-1333.

[5] Edvardsson, A Gustafsson and I Roos, Service portraits in service research: a critical review, International Journal of Service Industry Management, vol. 16(1), 2005, p. 107-121.

[6] Kindström, C. Kowalkowski and E. Sandberg, Enabling service innovation: A dynamic capabilities approach, Journal of Business Research, vol. 66(28), 2013, p. 1063-1073.

[7] Gremyr, L. Witell, N. Löfberg, et al., Understanding New Service Development and Service Innovation through Innovation Modes, Journal of Business \& Industrial Marketing, vol. 29(2), 2014, p. 4-4.

[8] S. Guo-qiang and L. Ji-ying, A Theoretical Framework of the Governance Mechanisms in Network Organization, Management Science and Engineering, ICMSE'06, 2006,p.947-951.

[9] X. Xue-mei, Empirical study on synergic innovative networks and innovation performance of SMEs, JOURNAL OF MANAGEMENT SCIENCES IN CHINA, vol. 13(8), 2010,p. 51-64. 